\title{
A Hybrid Link-TDMA MAC Protocol for Conventional and Radio over Fiber WLANs
}

\author{
Anastasios Valkanis, Petros Nicopolitidis $(\mathbb{D}$, and Georgios Papadimitriou \\ Department of Informatics, Aristotle University of Thessaloniki, Thessaloniki, Greece \\ Correspondence should be addressed to Petros Nicopolitidis; petros@csd.auth.gr
}

Received 24 November 2019; Revised 26 June 2020; Accepted 13 July 2020; Published 4 August 2020

Academic Editor: Vicente Casares-Giner

Copyright (c) 2020 Anastasios Valkanis et al. This is an open access article distributed under the Creative Commons Attribution License, which permits unrestricted use, distribution, and reproduction in any medium, provided the original work is properly cited.

\begin{abstract}
During the last two decades, the IEEE 8201.11 family has multiplied data rate transmissions in wireless local area networks (WLANs). This progress is based on the adoption of new technologies concerning physical layer (PHY), combined with the enhancements in the medium access control (MAC) layer. Radio over Fiber (RoF) is a technology which can further extend this progress, combining the benefits of optical fiber and wireless networks. The current IEEE 802.11 MAC protocol is contention based, which leads to low utilization of the RoF PHY data rate and on the other hand cannot handle the effects of long propagation delays in RoF networks. In this paper, we present a new Hybrid Link Time Division Multiple Access (HL-TDMA) MAC protocol which addresses the above shortcomings and enhances the performance of both conventional and RoF WLANs. Then, through simulation, we evaluate the performance of the proposed protocol, which is shown to be superior to relevant RoF WLAN protocols in the literature.
\end{abstract}

\section{Introduction}

The IEEE 802.11 standards have simplified the way that billions of users and devices connect to the internet. The reason behind the enormous adoption of this technology is the benefits it offers, including increased mobility of users, cost effective set up for hard to wire locations and reduced cost of ownership. The IEEE 802.11 Working Group has a structured method of introducing new technologies when a gap is identified in the current standard, leading to a long history of innovation as well as delivering both new technologies to PHY layer and enhancements to MAC layer in terms of security and quality of service.

The existing MAC protocol is still based on the distributed coordination function (DCF). However, DCF is contention based which leads to low utilization of the offered PHY data rate. Specifically, the new 802.11ac amendment can provide a maximum PHY data rate close to $7 \mathrm{Gbps}$ and only a maximum MAC data rate of $500 \mathrm{Mbps}$ for a single user scenario or $1 \mathrm{Gbp}$ for multiuser scenario [1]. Another issue is that DCF provides all nodes, including mobile station
(MS) and access point (AP), with the same channel access opportunities. However, in real situations, downlink- (DL-) offered load is much heavier than the uplink- (UL-) offered load causing unfairness problems between links [2].

RoF is a technology which can assist the evolution of Wi-Fi networks. In this kind of networks, a central station (CS) generates DL radio signals which are converted to optical ones by electrical to optical converters. Then, the optical signals are transferred through optical fiber and converted back to radio signals by optical to electrical converters before reaching remote antenna units (RAU) and finally transmitted to MS. The inverse procedure takes place for the UL packets from MS to CS as shown in Figure 1.

The main advantage of the $802.11 \mathrm{RoF}$ architecture is the combination of flexibility, low complexity, and mobility offered by wireless networks with the capacity and transparency of optical fiber networks [3]. Other advantages are the concentration of complexity in CS, allowing the use of simpler RAUs, enhanced coverage, and limitation of air propagation and transmission power, since the RAUs can be placed closer to mobile stations [4]. Furthermore, RoF 


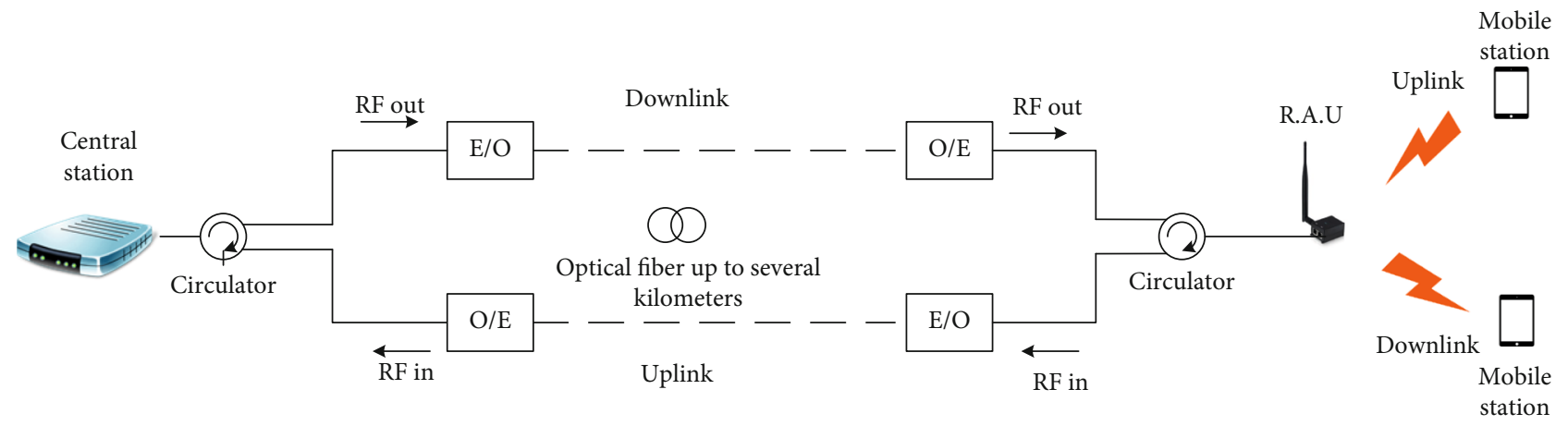

Figure 1: Architecture of 802.11 Radio over Fiber network.

technology is described as the ideal solution for centralized or cloud-based radio access networks, capable of serving the stringent demands of future applications, like tactile internet, in terms of reliability, data rate and latency $[5,6]$.

However, a significant problem arises when using IEEE 802.11 over RoF. The 802.11 MAC protocol is designed for propagation delay less than $1 \mu \mathrm{sec}$, whereas the injected delay to RoF network is $5 \mu \mathrm{sec}$ per kilometer of fiber. This fact degrades or even causes the overall performance of such a network to collapse completely due to increased number of collisions or acknowledgment (ACK) timeouts, when the existing IEEE 802.11 MAC protocol is used [7]. For this reason, it is essential to modify the current protocol, taking into consideration the effects that the extra propagation of fiber causes, in order for the protocol to be efficient and fully utilize the aforementioned advantages of 802.11 RoF networks.

In this paper, we present a hybrid HL-TDMA MAC protocol which on the one hand can successfully deal with the issues caused by the extra propagation delay of RoF 802.11 networks and on the other hand improves utilization of the medium. The proposed protocol is based on time sharing resources of network between UL and DL. In DL, there is no contention, whereas in uplink, MSs are contending for medium access. The HL-TDMA is an extension of the 802.11ac MAC protocol preserving its simplicity and requiring minor modification compared to the 802.11ac MAC protocol for the MS, since the slot time link allocation is fulfilled by interframe space (IFS) timing manipulation from the CS. Another contribution of this paper is that HL-TDMA improves not only RoF but also conventional 802.11 WLAN efficiency. In order to investigate the existing MAC protocol performance in 802.11 RoF network and evaluate our proposed protocol, we developed a RoF MAC simulator in MATLAB.

The rest of the paper is organized as follows. In Section 2, the behavior and shortcomings of the existing MAC protocol in RoF 802.11 networks is investigated both theoretically and via simulation results. Section 3 overviews related work on MAC protocols for this kind of networks. HL-TDMA and an analytical throughput model for it are presented in Section 4. In Section 5, we evaluate via simulation the performance of HL-TDMA protocol in comparison with the existing one for conventional networks as well as other proposed MAC protocols for RoF 802.11 networks, whereas conclusions and plans for future work are presented in Section 6.

\section{IEEE 802.11 MAC Protocol and Its Shortcomings in RoF}

The fundamental MAC technique of IEEE 802.11 standard is the DCF which employs Carrier Sense Multiple Access with Collision Avoidance (CSMA/CA) with binary exponential back off algorithm. It employs two mechanisms for reliable data delivery among stations, a basic one and an optional one named two-way and four-way hand shaking, respectively. The optional mechanism, known as the Request to Send/Clear to Send (RTS/CTS) is used to combat the hidden node problem but increases overhead due to additional control frames transmission. Moreover, when the basic data rates used for PHY preamble and header are much lower than the offered data rates, the four-way hand shaking mechanism loses its effectiveness to combat the hidden node problem due to the increased overhead [8]. The 802.11ac amendment for a $20 \mathrm{MHz}$ channel with one spatial stream can provide data rates up to $78 \mathrm{Mbps}$ when the basic rate used for compatibility issues with legacy devices is only $6.5 \mathrm{Mbps}$, which shows that the basic mechanism is more efficient than the optional one for most of the cases.

In the two-way hand shaking mechanism, a node which wishes to transmit a packet has to sense the medium for a period of arbitration interframe space (AIFS) plus a back off duration. If the medium is idle for the mentioned period, the node can access the medium and initiate a transmission. The back off duration is a pseudorandom time interval which is applied in order to avoid collisions between nodes wishing to access the medium. Its duration is calculated as

$$
\text { Backoff }=\operatorname{slot}(0, \mathrm{CW}) \times \text { slottime, }
$$

where $\operatorname{slot}(0, \mathrm{CW})$ is a pseudorandom integer from a uniform distribution in the interval $[0, \mathrm{CW}], \mathrm{CW}$ is the contention window size, and slottime is a fixed time value defined by protocol.

When the destination node receives the packet, it has to wait for short interframe space (SIFS) and transmit an acknowledgment (ACK) frame in order to accomplish the 
two-way handshaking procedure. If the ACK frame is not received after a period called ACK timeout, the packet is considered lost and the retransmission procedure is initiated. The CW size is limited in the interval shown below:

$$
\mathrm{CW}_{\min } \leq \mathrm{CW} \leq \mathrm{CW}_{\max }
$$

where $\mathrm{CW}_{\text {min }}$ is the minimum and $\mathrm{CW}_{\max }$ is the maximum CW size. After a successful transmission, CW is set to $\mathrm{CW}_{\text {min }}$, whereas after a retransmission, it is doubled, as shown below:

$$
\mathrm{CW}_{i}=\mathrm{CW}_{i-1} \cdot 2 \text {, }
$$

where $i$ is the retransmission attempt.

The above mechanism of existing MAC protocol is designed for a propagation delay of less than $1 \mu \mathrm{sec}$ when the injected delay to RoF network is $5 \mu \mathrm{sec}$ per kilometer of fiber. This fact leads to MAC performance degradation due to several reasons which will be analyzed in the rest of this section both theoretically and via simulation results.

2.1. Lack of Synchronization between CS and MS. There are two main differences between an RoF and a conventional WLAN. The first one is the existence of the optical part in a RoF WLAN and the second one is the distance between the MS and CS, where in a RoF WLAN can be up to several kilometers while in a conventional WLAN can be up to several meters. Due to the two mentioned differences, a synchronization problem between MS and CS arises, for the RoF WLANs using 802.11 MAC protocol, which it will be analyzed in the following section.

In the existing IEEE 802.11 MAC protocol, the nodes contending for the medium access are synchronized via the medium state. When a node accesses the medium and initiates a transmission, all the other nodes of the network are waiting for the end of transmission in order to access the medium following the timing rules defined by the protocol. The negligible propagation delay, due to the proximity between MS and CS and the lack of optical part existence, in a conventional WLAN allows the proper synchronization of nodes according to the medium state. In contrast, in an 802.11 RoF network when the CS accesses the medium, the MS cannot immediately sense the transmission and vice versa due to fiber propagation delay and the distance between them, which leads to lack of synchronization between CS and MSs.

In a conventional WLAN where all nodes are synchronized, a collision occurs when two or more nodes have the same back off slot value. Accordingly, a collision between a CS and a MS occurs when

$$
\mathrm{MS}_{\mathrm{SLOT}}=\mathrm{CS}_{\mathrm{SLOT}}
$$

where $\mathrm{CS}_{\mathrm{SLOT}}$ is the CS slot value and $\mathrm{MS}_{\mathrm{SLOT}}$ is the MS slot value. From (4), we have

$$
\mathrm{MS}_{\mathrm{SLOT}}-\mathrm{CS}_{\mathrm{SLOT}}=0 \text {, }
$$

which means that a collision between a MS and CS occurs when the difference between $\mathrm{CS}_{\mathrm{SLOT}}$ and $\mathrm{MS}_{\mathrm{SLOT}}$ is 0 or the corresponding space of values are

$$
\mathrm{MS}_{\mathrm{SLOT}}-\mathrm{CS}_{\mathrm{SLOT}} \in[0] \text {. }
$$

Since the slottime value is set to $9 \mu \mathrm{sec}$, the maximum propagation delay $T_{\text {SYN_MAX }}$ that a 802.11 RoF network can tolerate in order to have equal collision probability with a conventional one is [9]

$$
T_{\text {SYN_MAX }}=\frac{\text { slottime }}{2}=4.5 \mu \text { sec. }
$$

The $T_{\text {SYN_MAX }}$ corresponds to a fiber length $L_{\text {SYN_MAX }}$ of

$$
L_{\mathrm{SYN} \_\mathrm{MAX}}=\frac{T_{\mathrm{SYN} \_\mathrm{MAX}} \cdot c}{\mathrm{r} \_ \text {index }} \approx 900 \text { metres },
$$

where $c=3 \times 10^{8}$ is the speed of light and $r$ index $=1.5$ is the refractivity index of fiber. When the following condition is true,

$$
\text { Propagation }>T_{\text {SYN_MAX }}
$$

collision probabilities increase, since CS and MS frames collide due to lack of synchronization when,

$$
\left\{\begin{array}{l}
\mathrm{MS}_{\mathrm{SLOT}}=\mathrm{CS}_{\mathrm{SLOT}} \leq \mathrm{MS}_{\mathrm{SLOT}}+\lambda \\
\mathrm{CS}_{\mathrm{SLOT}}-\lambda \leq \mathrm{MS}_{\mathrm{SLOT}}=\mathrm{CS}_{\mathrm{SLOT}}
\end{array}\right.
$$

when the last ACK frame is DL or

$$
\left\{\begin{array}{l}
\mathrm{MS}_{\mathrm{SLOT}}-\lambda \leq \mathrm{CS}_{\mathrm{SLOT}}=\mathrm{MS}_{\mathrm{SLOT}} \\
\mathrm{CS}_{\mathrm{SLOT}}=\mathrm{MS}_{\mathrm{SLOT}} \leq \mathrm{CS}_{\mathrm{SLOT}}+\lambda
\end{array}\right.
$$

when the last ACK frame is UL, where $\lambda$ is a value which is dependent on propagation delay and is calculated as

$$
\lambda=\operatorname{ceil}\left(\frac{\text { Propagation }-T_{\text {SYN_MAX }}}{\text { slottime }}\right) \text {. }
$$

The corresponding to collision conditions (10) and (11) space values for $\mathrm{MS}_{\mathrm{SLOT}}-\mathrm{CS}_{\mathrm{SLOT}}$ are

$$
\begin{gathered}
\mathrm{MS}_{\mathrm{SLOT}}-\mathrm{CS}_{\mathrm{SLOT}} \in[0, \lambda], \\
\mathrm{MS}_{\mathrm{SLOT}}-\mathrm{CS}_{\mathrm{SLOT}} \in[-\lambda, 0],
\end{gathered}
$$

which shows that when propagation delay increases, the collision probability is also increasing because the $\lambda$ value is rising (12).

[10] shows that in a conventional WLAN, when a node's back off counter reaches zero, and the node accesses the medium, if the probability of each of the remaining nodes to transmit in the same slot is $\tau$, the collision probability $P_{\mathrm{COL}}$ is

$$
P_{\mathrm{COL}}=1-(1-\tau)^{n-1} \text {, }
$$


where $n$ is the number of network nodes. In a RoF WLAN, the collision probabilities $P_{\text {COL_DL }}$ due to desynchronization for a DL packet, if the probability of each of the MS to transmit in $\lambda$ slots is $\tau$, from (10) is

$$
P_{\mathrm{COL} \_\mathrm{DL}}=1-(1-\tau)^{m}
$$

where $m$ is the number of MSs. For an UL packet, the collision probabilities $P_{\text {COL_UL }}$ due to desynchronization, if the probability of CS to transmit in $\lambda$ slots is $\tau$, from (10) is

$$
P_{\mathrm{COL} \_\mathrm{UL}}=1-(1-\tau)^{1}=\tau
$$

since UL packets can collide only with a CS packet due to desynchronization. From Equations (15) and (16), we can see that the following condition is true

$$
P_{\mathrm{COL} \_\mathrm{UL}}=P_{\mathrm{COL} \_\mathrm{DL}}
$$

only when there is one MS in the network. When there are more than one MSs, the next condition is always true

$$
P_{\mathrm{COL} \_\mathrm{UL}}<P_{\mathrm{COL} \_\mathrm{DL}} \text {. }
$$

Equations (15) and (17) show that $P_{\mathrm{COL} \_\mathrm{DL}}$ rises when the number of MS increases and that desynchronization affects more the DL than the UL. An example in Figure 2 shows a scenario where in a conventional WLAN there is no collision when in a RoF one there is due to desynchronization effect.

2.2. Interframe Space Mechanism Colapse. The mechanism which provides a medium access priority in a conventional WLAN is the IFS. When a node receives a packet, it must reply immediately and not contend with other nodes for the medium access. For this reason, instead of waiting for an AIFS duration, it waits for a SIFS duration where SIFS $<$ AIFS. In an 802.11 RoF network, the IFS mechanism collapses for the UL due to the extra propagation delay of fiber (Figure 3). When a MS sends a packet, it is waiting for the ACK frame reply from the CS, while at the same time the other MSs are initiating the medium access procedure waiting for an AIFS plus a back off duration.

When the following condition is true,

$$
\text { AIFS }+ \text { Backoff }<\text { SIFS }+2 \cdot \text { Propagation, }
$$

the other MSs erroneously consider the medium as idle and initiate their transmission. So, the MS packet which will access the medium will collide with the delayed ACK frame from the CS. The maximum propagation delay $T_{\text {IFS_MAX }}$ that a 802.11 RoF network can tolerate in order for the IFS mechanism to work properly is

$$
T_{\text {IFS_MAX }}=\frac{\text { AIFS }+\min (\text { Backoff })-\text { SIFS }}{2}=18 \mu \mathrm{sec},
$$

where $\mathrm{AIFS}=43 \mu \mathrm{sec} \min ($ Backoff $)=9 \mu \mathrm{sec}$ and SIFS $=$
$16 \mu$ sec. The $T_{\text {IFS_MAX }}$ corresponds to a fiber length L_IFS_MAX of

$$
L_{\text {IFS_MAX }}=\frac{T_{\text {IFS_MAX }} \cdot c}{\text { r_index }} \approx 3600 \text { metres. }
$$

2.3. Acknowledgment Timeout. The 802.11 protocol defines an ACK timeout value for the reception of ACK frames. This value is not constant and varies depending on each vendor. In an 802.11 RoF network, when the fiber delay exceeds a certain value, the performance collapses due to ACK timeouts. The max tolerable propagation delay $T_{\text {ACK_MAX }}$ value is

$$
T_{\text {ACK_MAX }}=\frac{\text { ACK_timeout-SIFS }-T_{\text {ACK }}}{2},
$$

where ACK_timeout is set to $300 \mu \mathrm{sec}$ for our simulation scenarios, $\mathrm{SIFS}=16 \mu \mathrm{sec}$ and $T_{\mathrm{ACK}}$ is the duration of $\mathrm{ACK}$ frame. The $T_{\mathrm{ACK}}$ is calculated as

$$
\begin{aligned}
T_{\mathrm{ACK}} & =\mathrm{PHY}_{\mathrm{HEAD}}+\mathrm{PHY}_{\mathrm{PRE}}+\operatorname{ceil}\left(\frac{\text { ACK_size }}{\text { data bits/sym }}\right) \cdot \text { sym dur } \\
& =64 \mu \mathrm{sec},
\end{aligned}
$$

using the simulation values from Table 1. From (22), the $T_{\text {ACK_MAX }}=110 \mu \mathrm{sec}$ which corresponds to a fiber length L_ACK_MAX of

$$
L_{\text {ACK_MAX }}=\frac{T_{\text {ACK_MAX }} \cdot c}{\text { r_index }} \approx 22000 \text { metres. }
$$

2.4. Simulation Results. The simulation scenarios include an 802.11 ac RoF network using the existing basic two way handshaking MAC protocol which is more efficient than RTS/CTS with fiber length up to 22 kilometers. Also we have scenarios with 3,5,10 and $20 \mathrm{MS}$ associated to the CS. All the stations use the fixed Modulation and Coding Scheme number four (MCS4), and the rest simulation parameters are shown in Table 1. The traffic is saturated bidirectional User Datagram Protocol (UDP) which means that all MS and the CS have always packets in their buffer to send.

In Figure 4, we see the overall throughput performance of the network. A gradual decrease in throughput is observed as the fiber length increases due to extra fiber propagation delay. At $22 \mathrm{~km}$ of fiber length, we observe the total collapse of throughput as a result of ACK timeout and this value agrees with Equation (24) of the theoretical analysis. Also, we can see a negative slope after about $3 \mathrm{~km}$ of fiber length which is getting more negative as the number of MSs increases. This is explained by the IFS mechanism collapse and agrees with the value given by Equation (21).

In Figure 5, which illustrates the DL throughput performance of the network, we notice a sudden fall in DL throughput when the fiber length is longer than 900 meters due to desynchronization between CS and MS, which agrees with the value given by Equation (8). In contrast, a rising of throughput after $3 \mathrm{~km}$ of fiber length can be seen. This is 


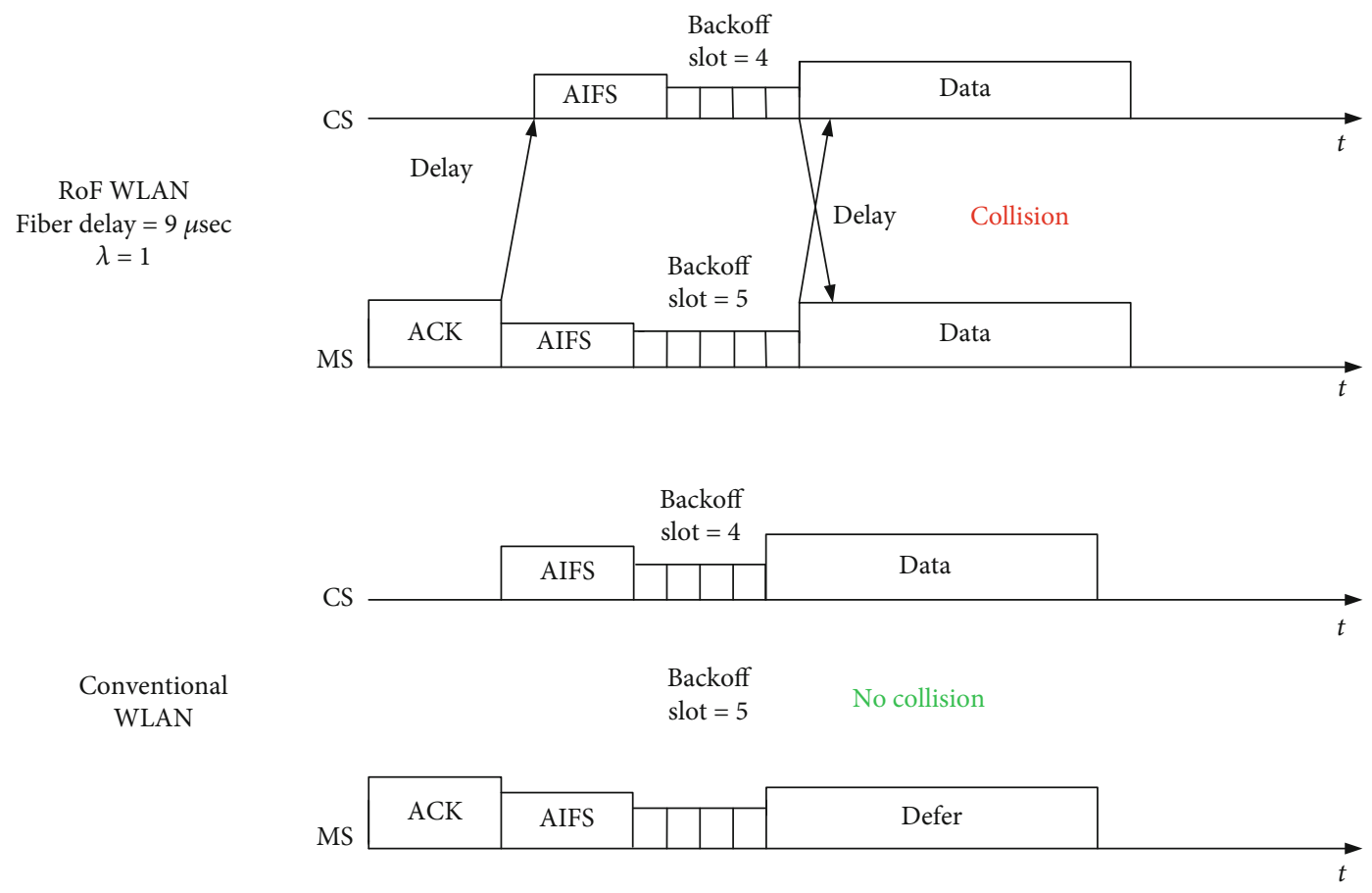

Figure 2: Desynchronization effect in a RoF WLAN.

ROF WLAN

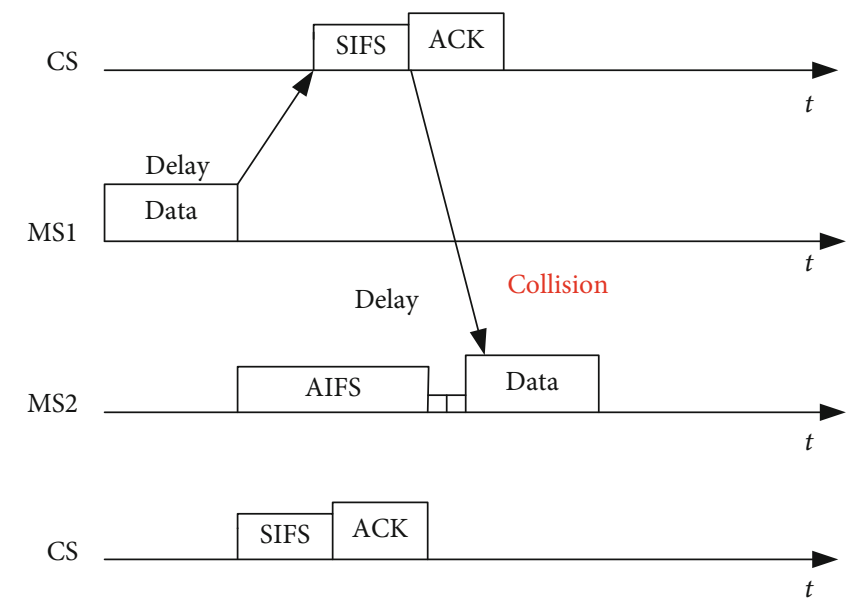

Conventional WLAN

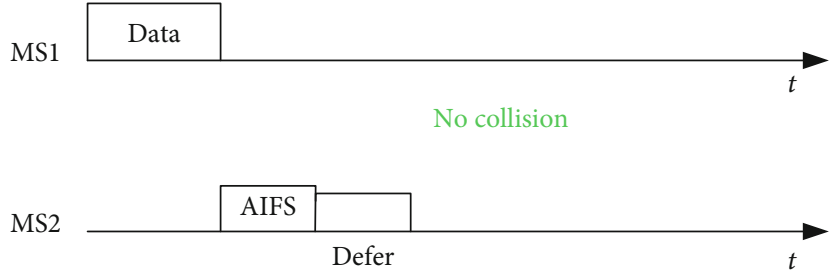

FIgURE 3: Interframe space mechanism collapse effect in a RoF WLAN.

explained by the IFS mechanism collapse for the UL which increases the CW for the MS and thus offers more transmission opportunities to the CS.

In Figure 6, we observe the UL throughput performance of the network. First, we can see that desynchronization does not severally affect the UL performance after 900 meters of fiber length due to low collision probability as shown in Equation (18); on the contrary, we can see the impact of IFS mechanism collapse which degrades the UL performance after $3.6 \mathrm{~km}$ of fiber length. 
TABLE 1: Simulation parameters.

\begin{tabular}{lc}
\hline Parameter & Value \\
\hline Physical data rate & MCS0-MCS9 \\
Basic physical data rate & MCS0 \\
Channel bandwidth & $20 \mathrm{MHz}$ \\
Antenna type & SISO \\
OFDM guard interval & Long \\
Acknowledgment timeout & $300 \mu \mathrm{sec}$ \\
Retry limit & 7 \\
CW min & 15 \\
CW max & 1023 \\
SIFS & $16 \mu \mathrm{sec}$ \\
AIFS & Best effort $43 \mu \mathrm{sec}$ \\
Slot time & $9 \mu \mathrm{sec}$ \\
HL-TDMA link slot duration & $3 \mathrm{msec}$ \\
Air propagation delay & $3 \mathrm{nsec}$ \\
Optical fiber length & $0-22 \mathrm{~km}$ \\
MAC payload & $1500 \mathrm{bytes}$ \\
Traffic & Saturated UDP \\
PHY preamble & $20 \mu \mathrm{sec}$ \\
PHY header & $20 \mu \mathrm{sec}$ \\
MAC header & $34 \mathrm{bytes}$ \\
Number of mobile stations & $1-20$ \\
Simulation time & $10 \mathrm{sec}$ \\
\hline
\end{tabular}

\section{Related Work}

A considerable amount of work has been done studying the performance of IEEE 802.11 existing MAC protocol in a RoF environment and on proposing new ones. In [11], an analysis on the feasibility of applying RoF techniques to the IEEE 802.11 MAC protocol is presented. An experimental investigation on throughput performance and hidden node problem is carried out in [12]. An analytical model for RoF WLANs is presented in [13], while the performance of IEEE 802.11n aggregation mechanism in RoF systems is investigated in [14]. Also, MAC protocols in RoF implementations at the $60 \mathrm{GHz}$ wireless domain are presented in $[15,16]$.

In order to overcome the negative effects of fiber's propagation delay in RoF WLANs, a slot adaption mechanism is proposed in [9]. This mechanism defines a $T_{\text {SYN_MAX }}$ propagation delay from Equation (7), when the following condition is true

$$
T_{\text {P_PROP }}>T_{\text {SYN_MAX }},
$$

slottime is adapted by adding the time taken by the fiber link propagation delay as

$$
\text { Slottime }=\text { Standard_slottime }+2 \times\left(T_{\text {P_PROP }}-T_{\text {SYN_MAX }}\right) \text {, }
$$

where $T_{\text {P_PROP }}$ is the fiber propagation delay. While the slottime adaption mechanism eliminates the phenomena of desynchronization and IFS mechanism collapse, at the same time, the overhead increases dramatically, thus reducing its overall efficiency. Another issue with this mechanism is the fairness between coexisting WLANs with the one using adapted slottime and the other using fixed slottime, in which case the fixed slottime WLAN nodes have more transmission opportunities.

Another MAC protocol, using the four-way hand shaking mechanism, for RoF WLANs is proposed in [17]. It uses a piggyback access (PBA) scheme from the CS in order to solve the desynchronization problem. This way there is no contention between CS and MSs, since instead of using the back off procedure for medium access, the CS sends its data packet after SIFS time from its ACK frame. This protocol also defines an $\alpha$ parameter $(0 \leq \alpha \leq 1)$ for controlling the transmission opportunities of the CS and balancing the throughput of the UL and DL. The protocol also eliminates the IFS mechanism collapse by employing an extended Network Allocation Vector (NAV) time on the CS Clear to Send (CTS) frame. The drawbacks of this protocol are the increased overhead due to the four-way hand shacking mechanism and that the CS is being unable to transmit a frame when there is no UL transmission.

A comprehensive study of hybrid CSMA/CA-TDMA MAC protocols are presented in [18]. All the suggested hybrid protocols are based on two common principles. The first one is the switching between the CSMA/CA and TDMA mode according to network condition and the second one is the effective time slot allocation between MS by the CS. However these facts increase the complexity of the MAC protocol in contrast with HL-TDMA which TDMA is used only for the separation of UL and DL transmissions.

\section{Hybrid Link-TDMA mac Protocol}

In order to eliminate the negative effects of existing MAC protocol in a RoF network, we propose the HL-TDMA protocol which is an extension of the existing 802.11ac MAC protocol. It is based on time sharing of network resources between UL and DL. The CS defines the timeslot duration for DL and UL according to the network condition and traffic. This is achieved artificially, without informing MSs, by IFS manipulation from CS. The new protocol requires a minor modification for the MSs compared to the existing one and all the complexity is concentrated in the CS. The fiber delay propagation $T_{\text {FIBER_PROP }}$ has to be estimated by CS in order to calculate a new IFS called Radio over Fiber IFS (RoFIFS). $T_{\text {FIBER_PROP }}$ can be estimated by measuring the time interval between a data frame sent from CS and an ACK frame sent from a MS. The RoFIFS value is calculated as

$$
\text { RoFIFS }=2 \cdot T_{\text {FIBER_PROP }}+\text { AIFS } .
$$

During the association stage every MS is informed for the RoFIFS value by the CS. The HL-TDMA can be used also in a conventional WLAN where there is no fiber propagation delay and RoFIFS = AIFS from Equation (27). 


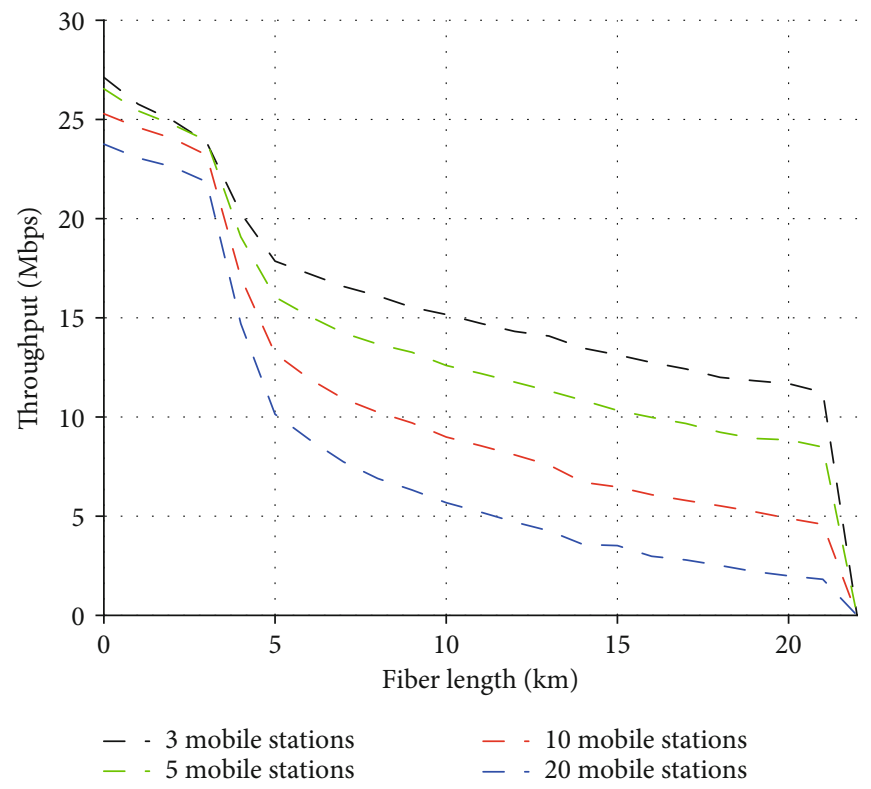

FIgURE 4: Aggregate throughput performance of the existing IEEE 802.11 MAC protocol in RoF WLAN.

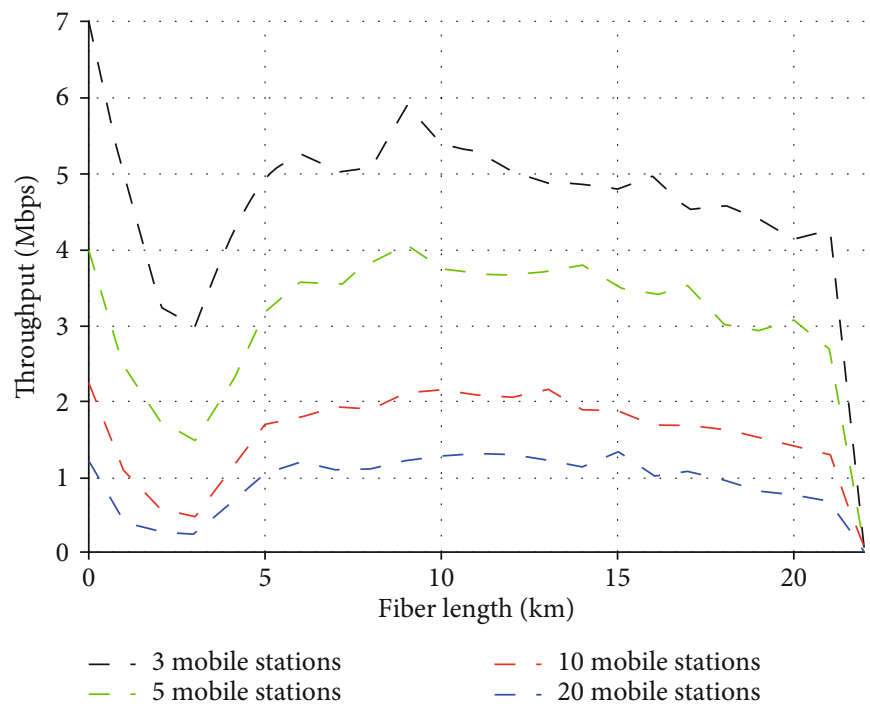

FIgURE 5: Downlink throughput performance of the existing IEEE 802.11 MAC protocol in RoF WLAN.

4.1. Central Station Medium Access Rules. As mentioned above, the CS defines two states for the network; the ppload-state (UL-state) and the download-state (DL-state) as shown in Figure 7. Two policies are employed for the switching between the two states. The first one distributes equally network resources among all stations (mobile and central), which results a ratio of 1: number of MSs allocated timeslots between DL and UL. The second employed policy, defines an equal distribution of network resources between UL and DL which results a ratio of $1: 1$ timeslots between links.

In the DL-state, the CS accesses the medium without using the DCF (Figure 8(b)). When an ACK frame is received from a MS, it waits for a SIFS duration and immediately accesses the medium if the next condition is true:

$$
\text { DL_State } \text { TIMER }>0 \text {, }
$$

or remains idle and sets the network to UL-state when Equa-

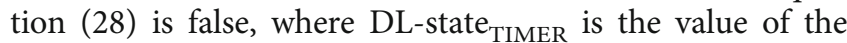
remaining DL-state time. In the UL-state, when the CS receives a data frame from a MS, it waits for a SIFS duration and remains idle after sending an ACK frame if the next condition is true.

$$
\text { UL_State }_{\text {TIMER }}>0 \text {, }
$$




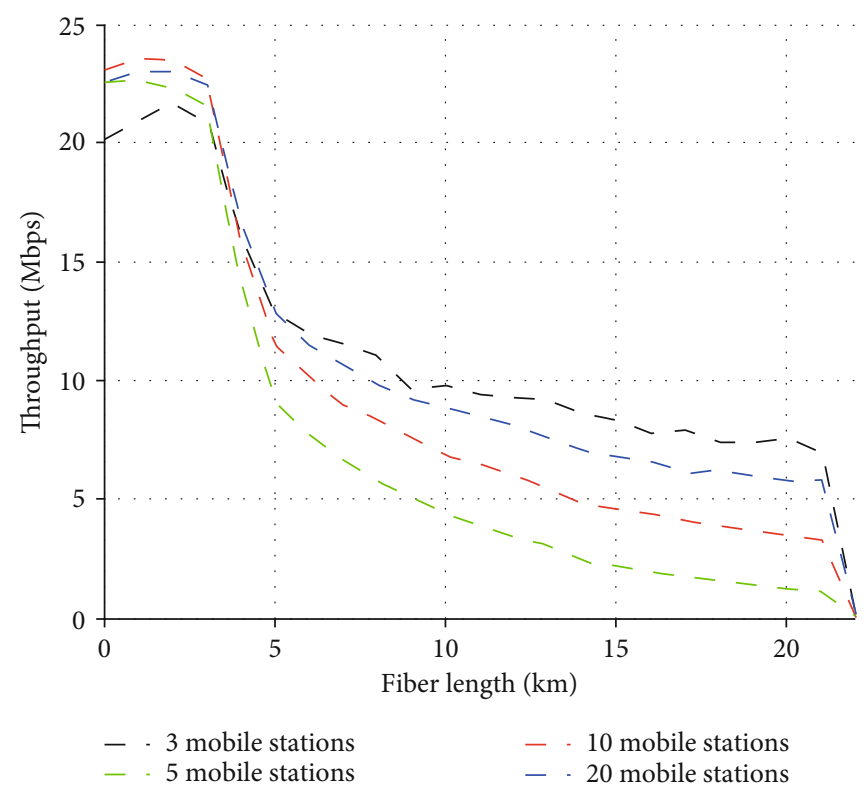

FIgURE 6: Uplink throughput performance of the existing IEEE 802.11 MAC protocol in RoF WLAN.

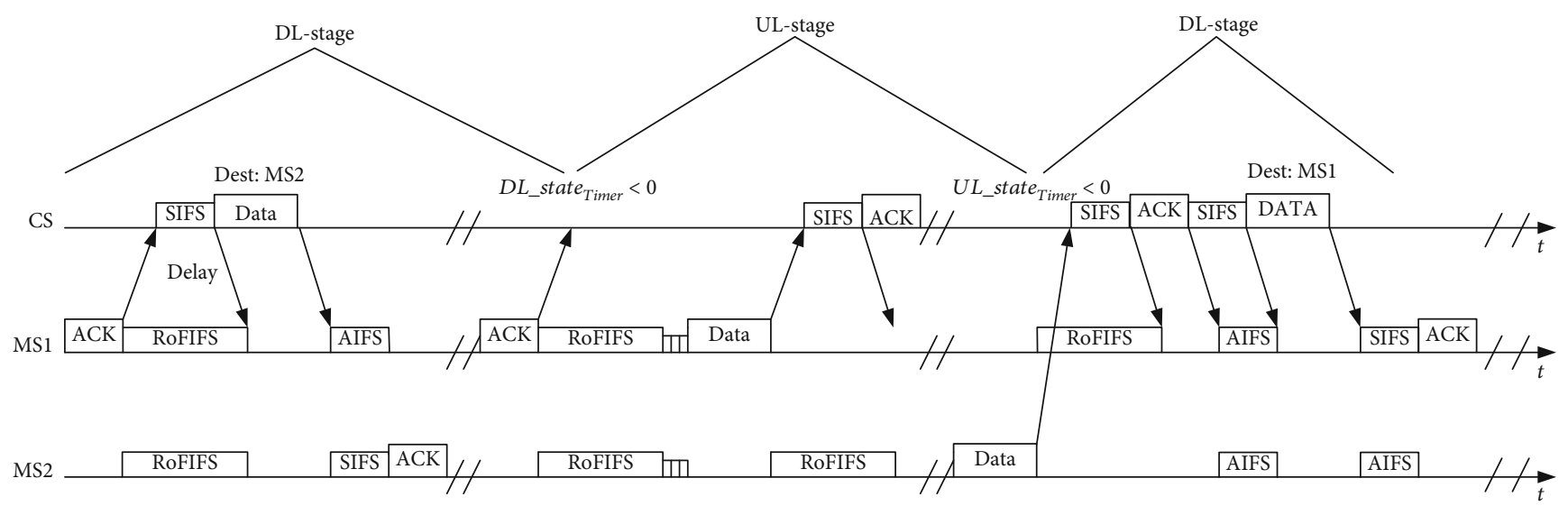

Figure 7: Download and upload stages of L-TDMA.

or sets network state to DL-state and waits for a SIFS duration after ACK frame before transmitting a data frame when Equation (29) is false, where UL-state TIMER is the value of the remaining UL-state time.

The use of the above medium access rules leads to the lack of contention between CS and MSs which in turn eliminates the desynchronization effect shown in a RoF WLAN using the existing MAC protocol.

4.2. Mobile Station Medium Access Rules. The HL-TDMA medium access rules for the MSs are based on the existing IEEE802.11 MAC protocol. The differences from the existing one are that there is no contention between MSs and CS and the utilization of a new IFS called RoFIFS (Figure 8(a)).

In IEEE802.11ac, the very high-throughput (VHT) portion of the VHT format preamble begins with the VHT Short Training Field (VHT-STF). VHT-STF contains a 6-bit information called Group ID whose main utility is for multiuser
(MU) packets [19]. When a single-user (SU) packet is sent to AP, the Group ID is set to 0 , if SU packet destination is a MS is set to 63 and if the packet is a MU one, the value is set between 1 and 62. Another usage of Group ID is the identification of packets link type; when Group ID is set to 0 , the packet is UL when in all other cases it is DL.

HL-TDMA defines that when a MS has a packet to send and senses a transmission, it defers its forthcoming transmission until the medium is idle. Utilizing the Group ID information, if the received packet is DL, it uses the same medium access rules with the existing MAC protocol but when the packet is UL, instead of waiting for AIFS duration before initiating back off procedure it is waiting for RoFIFS duration.

By using the above medium access rule, the ACK frame from CS is protected from a collision with a data frame transmission from another MS and thus eliminating the IFS mechanism collapse shown in a RoF WLAN using the existing MAC protocol. 


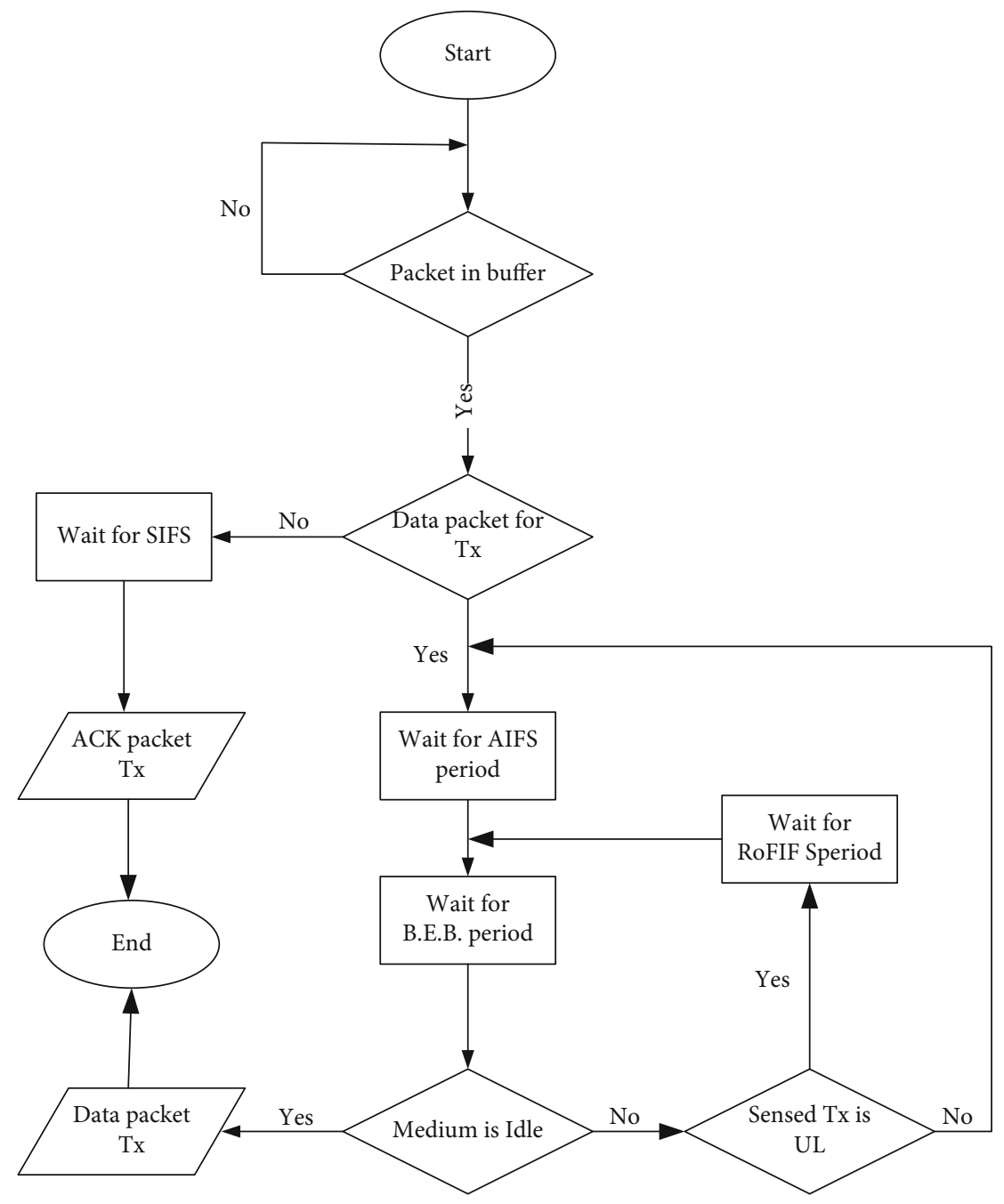

(a)

FIgURe 8: Continued. 


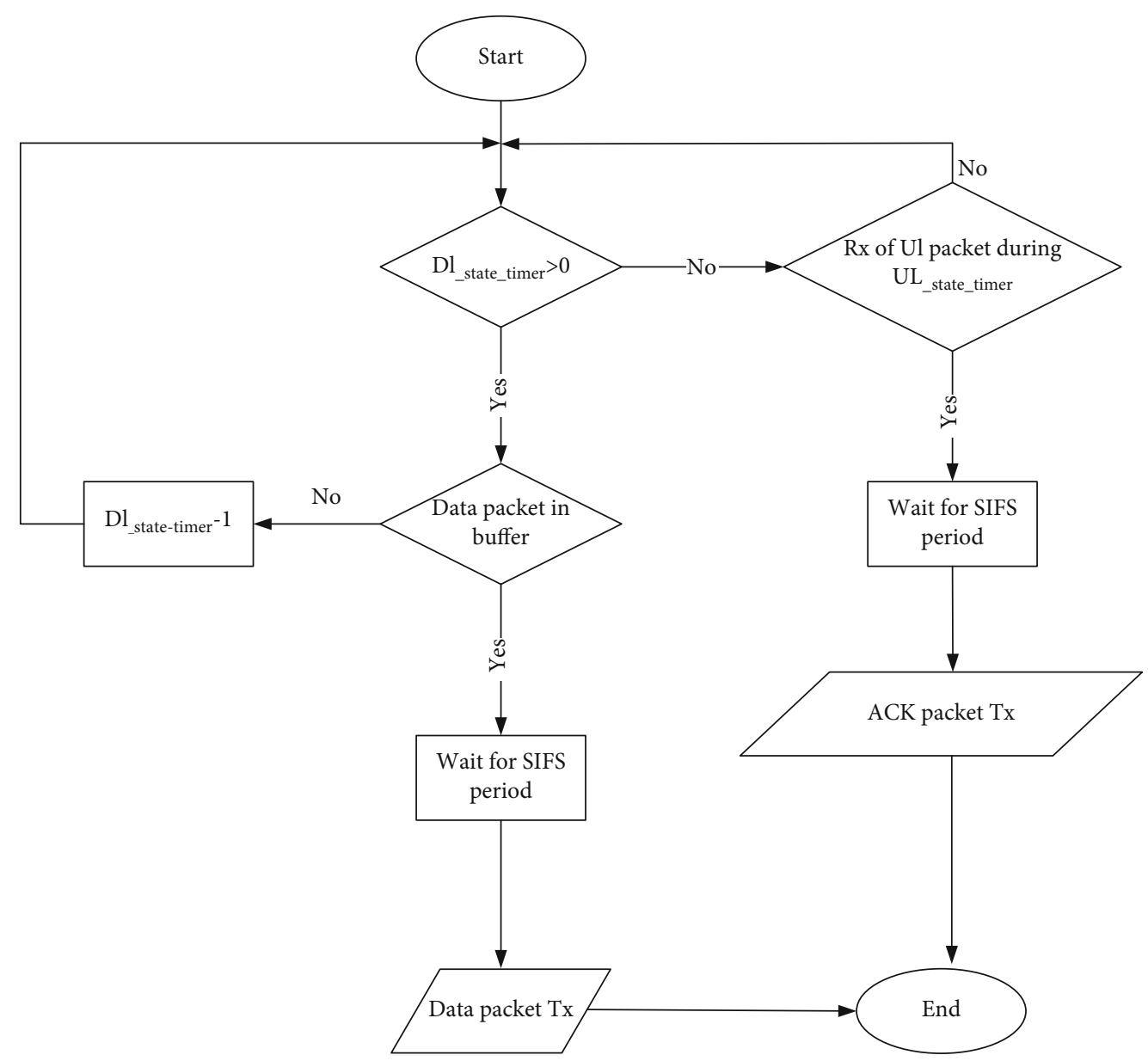

(b)

FIGURE 8: Medium access procedure flowchart for (a) mobile stations and (b) central station.

4.3. Analytical Throughput Model. The analytical throughput model for HL-TDMA we provide is based on Bianchi's well known analytical model for WLAN saturated UDP traffic using the existing MAC protocol [10]. Bianchi's model shows that the probability $\tau$ that a node transmits in a randomly chosen slot is

$$
\tau=\frac{2(1-2 p)}{(1-2 p)(W+1)+p W\left(1-(2 p)^{m}\right)},
$$

where $p$ is the conditional collision probability, $W$ is the contention window size, and $m$ is the maximum back off stage.

The conditional collision probability $\mathrm{p}$ is expressed as

$$
p=1-(1-\tau)^{n-1}
$$

where $n$ is the number of WLAN nodes. Equations (30) and (31) represent a nonlinear system with two unknowns $\tau$ and $p$ which can be solved using numerical techniques. The $P_{t r}$ probability that there is at least one transmission in the considered timeslot is

$$
P_{t r}=1-(1-\tau)^{n}
$$

and the probability $P_{s}$ that a transmission is successful is

$$
P_{s}=\frac{n \tau(1-\tau)^{n-1}}{P_{t r}}
$$

The normalized throughput $S$ is

$$
S=\frac{P_{s} P_{t r} E[P]}{\left(1-P_{t r}\right) \sigma+P_{t r} P_{s} T_{s}+P_{t r}\left(1-P_{s}\right) T_{c}},
$$

where $E[P]$ is the time needed for a packet transmission, having the average payload size employed in simulation, $T_{s}$ is the average time the channel is sensed busy due to successful transmission, $T_{c}$ is the average time the channel is sensed busy during a collision, and $\sigma$ is the slot time value. The $T_{s}$ and $T_{c}$ values for the basic two-way handshake scheme are

$$
\begin{gathered}
T_{s}=H+E[P]+\mathrm{SIFS}+\delta+\mathrm{ACK}+\mathrm{DIFS}+\delta, \\
T_{c}=H+E[P]+\mathrm{DIFS}+\delta,
\end{gathered}
$$

where $H$ is the duration of physical layer header and $\delta$ is the propagation delay. 
Equations (31)-(35) are modified for our analytical model as follows. The collision probability $p$ from (31) is

$$
p^{\prime}=1-(1-\tau)^{n-2},
$$

since the CS is not contending for the medium access, for the same reason (32) and (33) are modified as

$$
\begin{gathered}
P_{t r}^{\prime}=1-(1-\tau)^{n-1}, \\
P_{s}^{\prime}=\frac{n \tau(1-\tau)^{n-2}}{P_{t r}} .
\end{gathered}
$$

The average time the channel is sensed busy due to successful transmission for the UL $T_{\mathrm{UL}_{-} \mathrm{s}}$ is

$$
T_{\mathrm{UL} \_s}=H+E[P]+\mathrm{SIFS}+2 \delta+2 \delta^{\prime}+\mathrm{ACK}+\mathrm{AIFS},
$$

the average time the channel is sensed busy during a collision for the UL $T_{\mathrm{UL}_{-} \mathrm{c}}$ is

$$
T_{\mathrm{UL}_{-} \mathrm{c}}=H+E[P]+\mathrm{RoFIFS}+\delta+\delta^{\prime},
$$

the average time the channel is sensed busy due to successful transmission for the DL $T_{\mathrm{DL}_{-} \mathrm{s}}$ is

$$
T_{\text {DL_s }}=H+E[P]+2 \mathrm{AIFS}+2 \delta+2 \delta^{\prime}+\mathrm{ACK},
$$

where $\delta$ is the fiber delay. Since there is no contention for the $\mathrm{DL}$, the average time the channel is sensed busy during a collision for the $\mathrm{DL}$ is $T_{\mathrm{DL}_{-} \mathrm{c}}=0$.

If $b$ and $c$ are the number of time slots assigned to DL and $\mathrm{UL}$, respectively, the normalized throughput $S_{\mathrm{L}-\mathrm{TDMA}}$ is a summation for the uplink throughput and the downlink throughput since there are different medium access rules for each link. From (29), with the mentioned modified factors adapted to HL-TDMA, the normalized throughput $S_{\mathrm{L}-\mathrm{TDMA}}$ is

$$
\begin{aligned}
S_{\mathrm{L}_{\_} \mathrm{TDMA}}= & \left(\frac{b}{b+c} \frac{E[P]}{T_{\mathrm{UL}_{\_} s}}\right) \\
& +\left(\frac{c}{b+c} \frac{P_{s}^{\prime} P_{t r}^{\prime} E[P]}{\left(1-P_{t r}^{\prime}\right) \sigma+P_{t r}^{\prime} P_{s}^{\prime} T_{\mathrm{UL}_{-} s}+P_{t r}^{\prime}\left(1-P_{s}^{\prime}\right) T_{\mathrm{UL}_{\_} c}}\right) .
\end{aligned}
$$

\section{Performance Evaluation}

In this section, we evaluate HL-TDMA performance in comparison with the PBA [17], the slot adaption [9] and the existing MAC protocols; for this reason, we developed a MAC simulator for conventional and RoF WLANs in MATLAB. Performance evaluation for HL-TDMA is made both by our analytical modeling as well as our simulation, and the two approaches are always shown to give the same results. The first three scenarios consider a RoF WLAN topology investigating throughput performance as a function of variable fiber length, a variable MCS used by the network nodes and a variable number of MSs associated with a CS. The

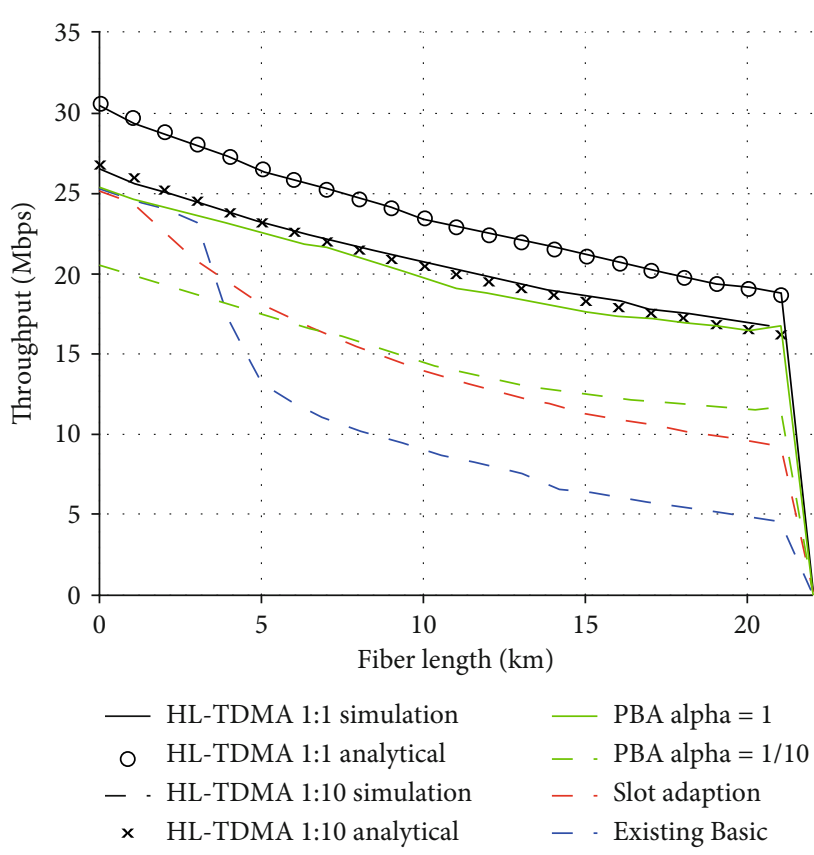

FIGURE 9: MAC protocols (UL+DL) throughput performance in a RoF WLAN with variable fiber length.

fourth scenario also considers a RoF WLAN topology and investigates the throughput ratio of DL and UL as a function of the number of MSs. The duration of both UL and DL time slots for the HL-TDMA is set to $3 \mathrm{msec}$. For the HL-TDMA and PBA we use two different resource allocation schemes for UL and DL, as follows: The ratio of time slots between DL and UL for the first HL-TDMA scheme is $1: 1$ which means that network resources are equally shared between links. For the first PBA resource allocation scheme the $\alpha$ value is set to 1 which balances the throughput of the UL and DL equally. The second scheme for HL-TDMA and PBA provides all nodes including CS with the same channel access opportunities. For this reason, the ratio of time slots between DL and UL for the HL-TDMA is set to 1:number of MSs and the $\alpha$ value for the PBA is set to $1 /$ number of MSs.

The first scenario includes a RoF WLAN with fiber length up to 22 kilometers, with 10 MSs associated to CS and all the nodes using MCS 7. Results are shown in Figure 9, which depict the superiority of L-TDMA for all fiber lengths. Additionally, the performance is better when the ratio between links is $1 / 1$ and $\alpha$ is set to 1 due to reduced DL overhead in both cases.

Figure 10 depicts the throughput performance of RoF WLAN in the second scenario with 10 MSs associated to CS when variable MCS is used by network nodes and fiber length is $10 \mathrm{~km}$. As expected, the throughput is increasing proportional to the offered PHY data rate increase for all the protocols. Also, it is revealed by the results that the performance difference between HL-TDMA and other protocols is increased when the PHY data rate is also increased due to reduced overhead that HL-TDMA employs compared to other schemes.

The third scenario for a RoF WLAN includes a variable number up to 20 of MSs associated to CS, the fiber length is 


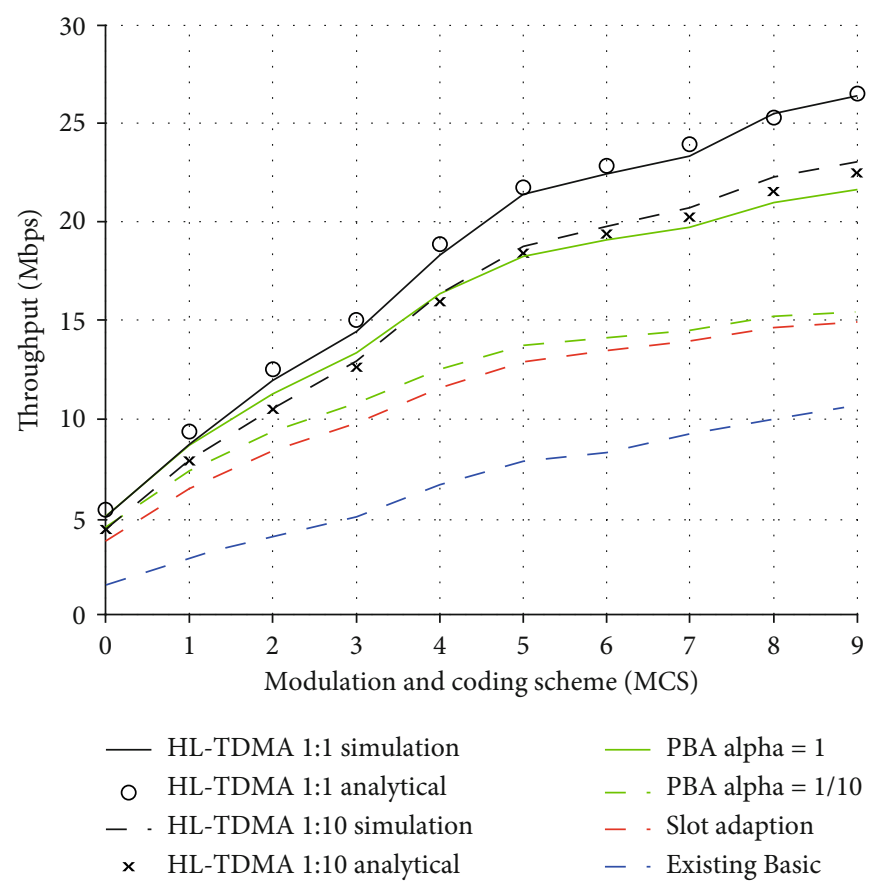

FIgURE 10: MAC $(\mathrm{UL}+\mathrm{DL})$ protocols throughput performance in a RoF WLAN with variable PHY data rate used by network nodes.

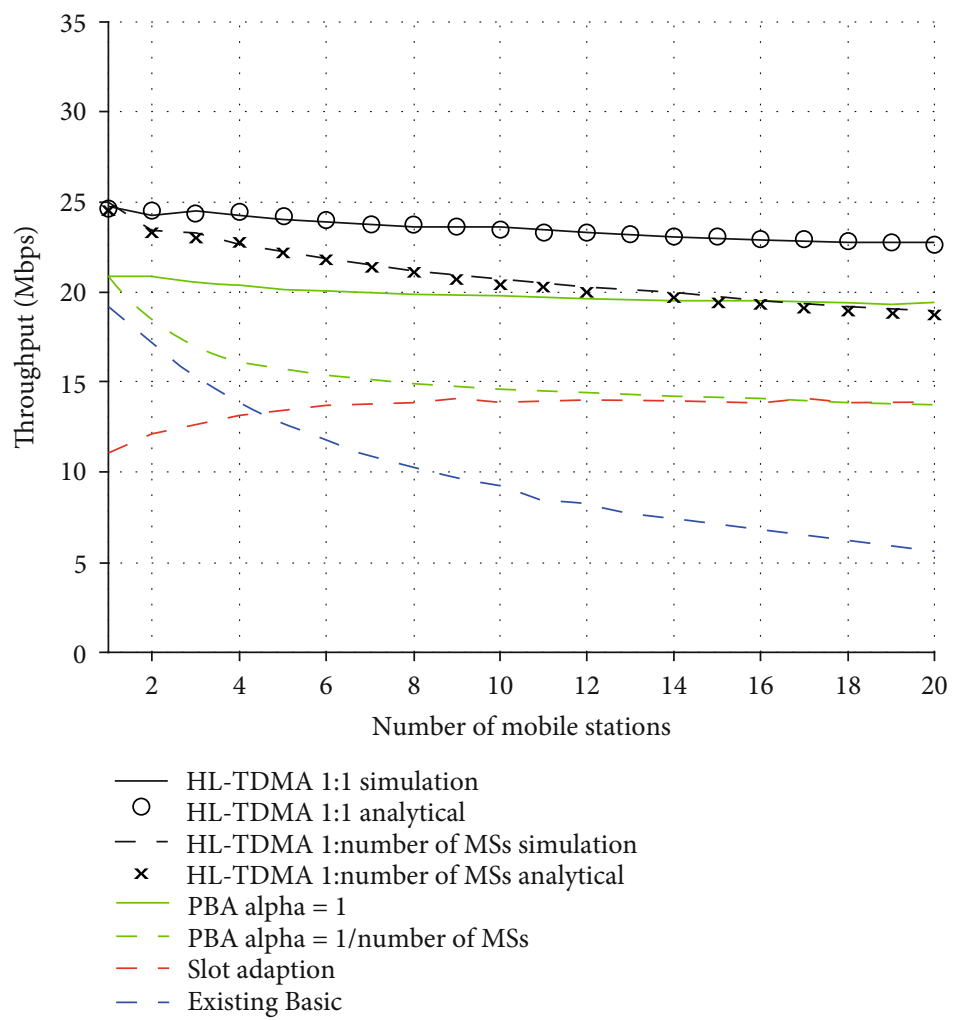

FIGURE 11: MAC protocols (UL + DL) throughput performance in a RoF WLAN with variable number of mobile stations.

10 kilometers and all the nodes using MCS 7. Results in Figure 11 show the HL-TDMA superiority against other protocols in all cases and the poor performance of the PBA when $\alpha$ is set to $1 /$ number of MSs and when the number of MSs is increased. The low variation in aggregate throughput, observed in this scenario for all the protocols, when the number of stations increase can be explained by the combination of two incidents. The first one is the increased slot utilization, 


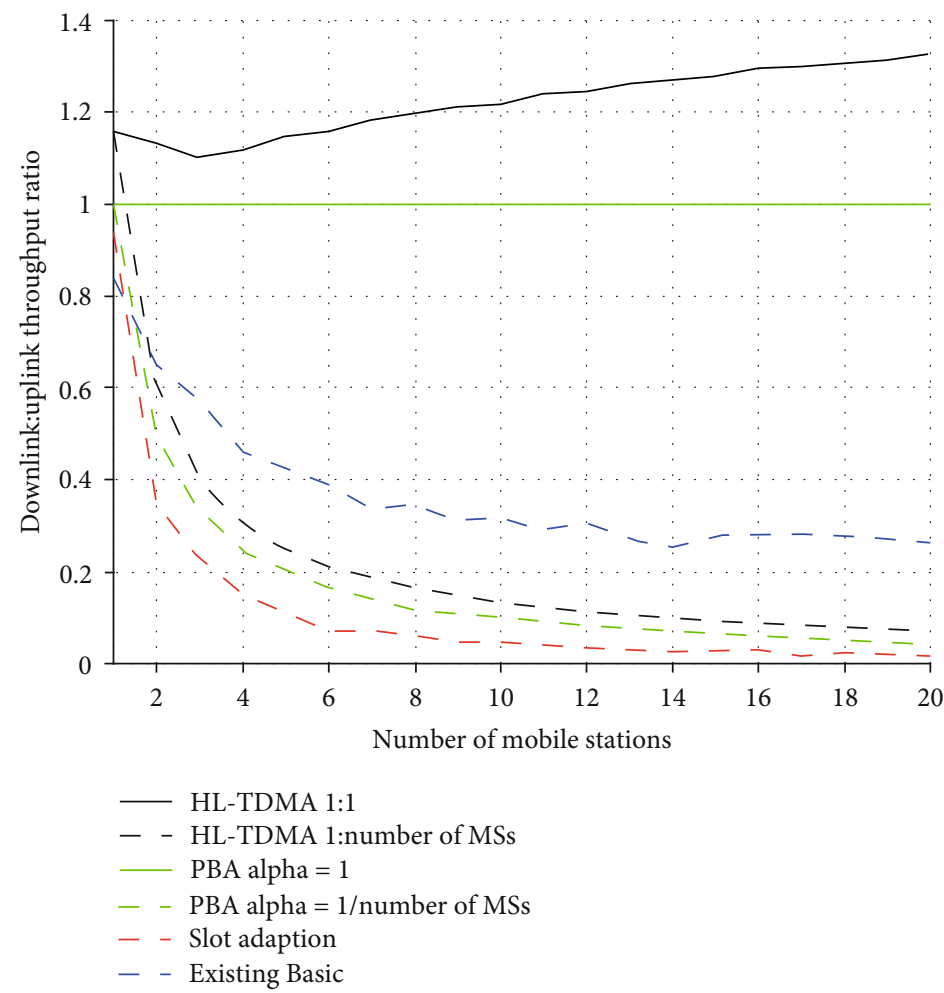

FIGURE 12: Throughput ratio between uplink and downlink for the MAC protocols with variable number of MSs.

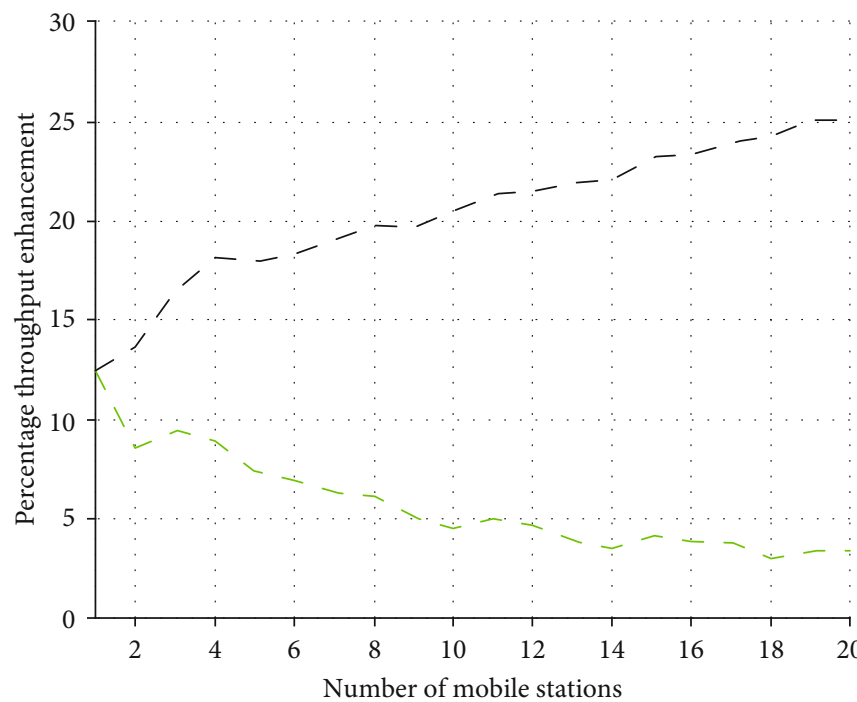

- - HL-TDMA shares time slots equally between UL and DL

HL-TDMA uses same channel access opportunities as the existing MAC protocol for the nodes

FIGURE 13: Percentage throughput enhancement of L-TDMA compared to existing MAC protocol in a conventional WLAN with variable number of MSs.

which affects positively the throughput performance, since there are more stations trying to access the medium and the second one is the increased probability of collisions, which affects negatively the throughput performance, since all the stations are contending for the medium. Also we can see the poor throughput performance of the slot adaption mech- anism even when the number of MS is small, this fact is explained by the increased overhead that slot adaption employs compared to other schemes.

The fourth scenario for a RoF WLAN has the same parameters as the third scenario Results in Figure 12 shows the impact of the resource allocation policies employed by 
HL-TDMA and PBA. The ratio of throughput between uplink and downlink follows the ratio of timeslots assigned for them from HL-TDMA and the $a$ value employed by PBA. The HL-TDMA link balancing is not as accurate as the PBA due to contention between MSs and increased overhead of the UL.

Finally, we also evaluate HL-TDMA MAC throughput percentage enhancement in comparison with the existing MAC protocol in a conventional single-hop radio WLAN. The simulation includes a variable number of up to $20 \mathrm{MSs}$ associated to AP, and a MCS 7 used by the network nodes. It is shown by the results in Figure 13 that HL-TDMA enhances the MAC efficiency in all cases. For the first HL-TDMA resources allocation scheme, where time slots are equally shared between UL and DL, enhancement is increased when the number of MSs is increased as well in contrast to the second one, which provides all nodes including CS with the same channel access opportunities as the existing MAC protocol, where enhancement is decreased when the number of MSs is increased due to increased UL overhead.

\section{Conclusions}

Combining the advantages of optical fiber and wireless networks, $802.11 \mathrm{RoF}$ is a promising technology. Based on our investigation of the behaviour of the existing MAC protocol in a RoF WLAN environment, theoretical analysis and simulation results show the feasibilities of such a deployment. Taking into consideration the pecuilarity of RoF enviroment we have proposed a hybrid MAC protocol called HL-TDMA. The proposed MAC protocol is based on the existing 802.11ac one and requires a minor modificaton for MSs compared to it. Another advantadge of HL-TDMA is the balance of network recources between links related to traffic conditions. This balance is adjusted by CS which allocates time slots for UL and DL. Simulation results show that HL-TDMA enhances the throughput performance in a RoF WLAN compared to the existing IEEE 802.11 MAC protocol one and other MAC protocols designed for RoF envionments. Moreover, simulation results reveal that the proposed protocol also enhances the throughput performance up to $25 \%$ compared to the existing MAC protocol in a conventional WLAN.

Our future works include the evaluation of HL-TDMA with various types of unsaturated traffic. Also we consider the design of an algorithm operating in the CS, which will allocate the time slots for UL and DL according to the network environment and traffic in order to optimize parameters including fairness, latency, and efficiency. Finally the effects of RoF and conventional WLAN coexistence are included in our future work.

\section{Data Availability}

Data can be provided upon request.

\section{Conflicts of Interest}

The authors declare that there are no conflicts of interest regarding the publication of this paper.

\section{References}

[1] E. H. Ong, J. Kneckt, O. Alanen, Z. Chang, T. Huovinen, and T. Nihtilä, "IEEE 802.11ac: Enhancements for very high throughput WLANs," in 2011 IEEE 22nd International Symposium on Personal, Indoor and Mobile Radio Communications, pp. 849-853, Toronto, ON, USA, September 2011.

[2] C. Wang, H. Lo, K. Liang, and C. Hsu, "A cross-layer design for per-flow and weighted fairness between uplink and downlink in WLANs," in 2010 IEEE International Conference on Communication Systems, pp. 421-425, Singapore, Singapore, November 2010.

[3] A. M. J. Koonen, M. G. Larrodé, A. Ng’oma et al., "Perspectives of radio over fiber technologies," in OFC/NFOEC 2008 - 2008 Conference on Optical Fiber Communication/National Fiber Optic Engineers Conference, San Diego, CA, USA, Febuary 2008.

[4] Y. Josse, B. Fracasso, and P. Pajusco, "Model for energy efficiency in radio over fiber distributed indoor antenna Wi-Fi network," in 2011 The 14th International Symposium on Wireless Personal Multimedia Communications (WPMC), pp. 1-5, Brest, France, October 2011.

[5] Y. Kim, G. Kim, and H. Lim, "Cloud-based Wi-Fi network using immediate ACK in uplink data transmissions," IEEE Access, vol. 6, pp. 37045-37054, 2018.

[6] M. Maier and B. P. Rimal, "Invited paper: the audacity of fiberwireless (FiWi) networks: revisited for clouds and cloudlets," China Communications, vol. 12, no. 8, pp. 33-45, 2015.

[7] Y. Fan, J. Li, K. Xu et al., "Performance analysis for IEEE 802.11 distributed coordination function in radio-over-fiberbased distributed antenna systems," Opt. Express, vol. 21, no. 18, pp. 20529-20543, 2013.

[8] I. Tinnirello, S. Choi, and Y. Kim, "Revisit of RTS/CTS exchange in high-speed IEEE 802.11 networks," in Sixth IEEE International Symposium on a World of Wireless Mobile and Multimedia Networks, pp. 240-248, Taormina-Giardini Naxos, Italy, June 2005.

[9] S. Deronne, V. Moeyaert, and S. Bette, "Analysis of the MAC performances in $802.11 \mathrm{~g}$ radio-over-fiber systems," in 2011 18th IEEE Symposium on Communications and Vehicular Technology in the Benelux (SCVT), pp. 1-5, Ghent, Belgium, November 2011.

[10] G. Bianchi, "Performance analysis of the IEEE 802.11 distributed coordination function," IEEE Journal on Selected Areas in Communications, vol. 18, no. 3, pp. 535-547, 2000.

[11] B. L. Dang and I. Niemegeers, "Analysis of IEEE 802.11 in radio over fiber home networks," in The IEEE Conference on Local Computer Networks 30th Anniversary (LCN'05) l, pp. 744-747, Sydney, NSW, Australia, November 2005.

[12] A. Das, M. Mjeku, A. Nkansah, and N. J. Gomes, "Effects on IEEE 802.11 MAC throughput in wireless LAN over fiber systems," Journal of Lightwave Technology, vol. 25, no. 11, pp. 3321-3328, 2007.

[13] B. Kalantari-Sabet, M. Mjeku, N. J. Gomes, and J. E. Mitchell, "Performance impairments in single-mode radio-over-fiber systems due to MAC constraints," Journal of Lightwave Technology, vol. 26, no. 15, pp. 2540-2548, 2008.

[14] S. Deronne, V. Moeyaert, and S. Bette, "WiFi transmission in radio-over-fiber systems: performance of the IEEE $802.11 \mathrm{n}$ aggregation mechanism," in 2013 17th International Conference on Optical Networking Design and Modeling (ONDM), pp. 167-172, Brest, France, April 2013. 
[15] A. Panagiotakis, P. Nicopolitidis, G. I. Papadimitriou, and P. G. Sarigiannidis, "Performance increase for highly-loaded RoF access networks," IEEE Communications Letters, vol. 19, no. 9, pp. 1628-1631, 2015.

[16] G. Vasileiou, G. I. Papadimitirou, P. Nicopolitidis, and P. G. Sarigiannidis, "An effective resource allocation medium access control protocol for radio- over-fiber access networks based on wavelength reuse," Computer Communications, vol. 88, pp. 45-56, 2016.

[17] T. Nishio, K. Funabiki, M. Morikura, K. Yamamoto, D. Murayama, and K. Nakahira, "MAC protocol for improving throughput and balancing Uplink/Downlink Throughput for wireless local area networks with long propagation delays," IECE Transactions on Communications, vol. E100.B, no. 5, pp. 874-883, 2017.

[18] B. Shrestha, E. Hossain, and K. W. Choi, "Distributed and centralized Hybrid CSMA/CA-TDMA schemes for single-hop wireless networks," IEEE Transactions on Wireless Communications, vol. 13, no. 7, pp. 4050-4065, 2014.

[19] "Wireless LAN medium access control MAC and physical layer PHY specifications: enhansments for very high throughput for operation in bands below $6 \mathrm{GHz}$ IEEE standard 802.11ac," 2012. 